\title{
IMPROVING AUTOMATIC EVALUATION OF MANDARIN PRONUNCIATION WITH SPEAKER ADAPTIVE TRAINING (SAT) AND MLLR SPEAKER ADAPTION
}

\author{
Chao Huang ${ }^{l}$, Feng Zhang ${ }^{I^{*}, 2}$ and Frank K. Soong ${ }^{1}$ \\ ${ }^{1}$ Microsoft Research Asia, Beijing \\ 2 iFlytek Speech Lab, University of Science and Technology of China, Hefei \\ zhangf@ustc.edu, \{chaoh, frankkps\}@ microsoft.com
}

\begin{abstract}
Automatic pronunciation evaluation (APE) can be implemented with a speech recognition model trained by standard, "golden" speakers. The pronunciation accuracy is then measured with the Goodness of Pronunciation (GOP) as reported in our earlier work [1]. In this paper, we investigate two main strategies for improving the evaluation: speaker adaptive training (SAT) for reducing the speaker-specific characteristics in model training and MLLR-based speaker adaptation in evaluation for reducing mismatch between the trained model and a testing speaker. Overall, the proposed strategies improve the correlation between evaluations made by APE and human experts from 0.69 to 0.76 , approaching the upper bound value of 0.78 among human expert evaluators. Additionally, APE also shows a consistency of 0.93 better than the consistency of 0.83 among human experts.
\end{abstract}

Index Terms-Speaker adaptation, speaker adaptive training (SAT), automatic pronunciation evaluation (APE), posterior probability (PP)

\section{INTRODUCTION}

Computer assisted language learning (CALL) has become more popular with the aid of the modern ASR technology. In a CALL system, it is critical to get an instantaneous, objective evaluation feedback on the learner's pronunciation quality.

Most APE studies are focused on the second language learning and evaluation. However, evaluating a native speaker's pronunciation quality is also very useful for correcting accented or inaccuarate pronunciations in a big country like China where many dialects and accents are spoken. The proficiency test of Mandarin called Putonghua Shuiping Ceshi (PSC) is an official standard test to evaluate a testee's oral Mandarin proficiency level. A certificate of PSC above a certain grade is required for many vocations in China, such as teachers, radio or TV announcers and government officials. It is both time-consuming and costly to employ human experts to grade a testee's pronunciation. Attempts have been launched to automate the evaluation procedure.

Many features like log-likelihood, recognition accuracy and posterior probability have been proposed as the Goodness of Pronunciation (GOP) measure [2,9]. A refined GOP based on the generalized segment posterior probability has been proposed and reported in our previous work [1]. Other algorithms such as the work in [6] also deliver a good evaluation performance. These

*Join the work as an intern at Microsoft Research Asia works are mostly focused on the selection of the better measures for APE.

In this paper, research on how to make a more canonical APE model is investigated. Speaker normalization in training and speaker adaptation in evaluation is adopted to improve the model canonicity. To do the evaluation, GOP based on posterior probability is applied both at syllable level and phone level. The results verify the validity of the proposed schemes in APE.

This paper is organized as follows. Section 2 presents the GOP measures and proposed improvement of APE evaluation model. In Section 3, the experimental database is described. The evaluation results and analysis are given in Section 4. Conclusions are drawn in Section 5.

\section{IMPROVE APE WITH SAT AND MLLR}

Before present the strategies to improve the model canonicity, we will first briefly review the GOP measure used in the paper.

\subsection{GOP measure}

To assess the pronunciation quality of a speaker, many measures have been proposed before. In this paper, posterior probability is chosen as the GOP measure since it is less affected by the changes in spectrum due to speaker specific characteristics or acoustic channel variations. It measures the phonetic quality more directly than other measures. We review it briefly here while leave the details to our work reported previously [1]

In an HMM-based speech recognizer, given an isolated syllable or phone acoustic observation sequence $O$ and its corresponding transcription $w_{i}$, GOP score based on $\log$ posterior probability (Log-PP) can be written as:

$$
G\left(w_{i} \mid O\right)=\log \left(\frac{p\left(O \mid w_{i}\right) p\left(w_{i}\right)}{\sum_{j=1}^{J} p\left(O \mid w_{j}\right) p\left(w_{j}\right)}\right)
$$

$w_{i}$ in Eq. (1) can be either a syllable or a phone. Given GOP scores at phone level, GOP at syllable level can also be calculated in other two ways: the averaged or weighted by their duration as shown in Eq. (2).

$$
G\left(s_{i} \mid O\right)=\frac{D_{i}}{D_{i}+D_{f}} G\left(p_{i} \mid O\right)+\frac{D_{f}}{D_{i}+D_{f}} G\left(p_{f} \mid O\right)
$$

where $D_{i}$ and $D_{f}$ are the duration of the phone $p_{i}$ and $p_{f}$ for syllable $s_{i}$ respectively (In Mandarin, each syllable normally consists of two phones, called initial and final). GOP scores for a speaker can be calculated as the sum of the GOPs at syllables or phone levels.

\subsection{Improved APE with SAT and MLLR}


To improve the performance of APE, in addition to searching for a better GOP measure, we also like to build a better evaluation model. In this paper, we will focus on strategies to improve the model canonicity. Two strategies are investigated. As we know, statistical models that used to calculate the GOP in APE are normally trained based on a large amount of utterances from many speakers. In other word, model consists of two main information, speaker-specific and phonetic-specific while the former is not expected since we care only the phonetic correctness or not in APE. To eliminate this effect, an approach of speaker adaptive training (SAT) based on constrained maximum likelihood linear regression (CMLLR) is used for normalizing the acoustic data during the training. During the evaluation, to reduce the mismatch between the "neutral" model and the testing data further, speaker adaptation based on maximum likelihood linear regression (MLLR) is used to adjust the neutral space to the target's space based on these correct phonetic pronunciations.

\subsubsection{Speaker normalization using SAT}

The parameters of Hidden Markov Model (HMM) are usually trained based on the data from a large number of speakers. In this way, the parameters are unavoidably affected by inter-speaker variability induced by the different characteristics of the speakers. It is not only one of the major causes of error in ASR, but also greatly affects the performance of APE. Speaker normalization attempts to reduce inter-speaker acoustic variability induced by the different characteristics of each speaker. SAT is proved to be an efficient approach to reduce such variability in speakerindependent (SI) model caused by variation among the speakers [3, 4]. With explicit modeling of speakers by linear transformations based on maximum likelihood criteria and applying inverse transformation back to training features from each speaker, SAT can obtain a more neutral model with less speaker variability. There are two main forms of SAT. One is the standard one [3] where the mean and variance transformations are unrelated to each other. The other is the constrained one (CMLLR) [4] where the transformation $A^{\prime}$ 'on the means $\mu$ and variances $\nu^{\prime}$ are required to have the same form, other than the bias $b^{\prime}$. Thus, the general form of CMLLR is

$$
\begin{aligned}
& \hat{\mu}=A^{\prime} \mu-b^{\prime} \\
& \hat{\Sigma}=A^{\prime} \sum A^{T}
\end{aligned}
$$

Specifically, CMLLR is a feature adaptation technique that estimates a set of linear transformation for the features. With its comparatively easy implementation, SAT based on CMLLR is used in the paper.

\subsubsection{Speaker adaptation using MLLR}

With SAT, we can obtain more compact or neutral model in training that reflected by smaller variance in the model. Given the testing features from target that includes both speaker-specific and phonetic-specific variations, adaptation is needed to reduce such mismatch further. Otherwise, target speaker may achieve the worse scores only due to the mismatch between the compact model after SAT and the speaker's characteristics. However, there is an important difference using speaker adaptation for ASR and APE. For ASR, speaker adaptation is used to reduce the mismatch as far as possible, no matter such mismatch is caused by the characteristics or the mispronunciation of the speaker. For APE, speaker adaptation should be adopted carefully to reduce the mismatch caused by the characteristics instead of the mispronunciation of the speaker. To achieve this purpose, MLLR setup with one or a few transformations can be competent in that it keeps the main characteristic of speaker while ignoring the pronunciation details.

\subsubsection{Combined schemes to improve APE}

To testify the proposed strategies, we design the following combined schemes in our experiment to verify the MLLR and SAT respectively and jointly.

\begin{tabular}{|c|c|c|c|}
\hline $\begin{array}{l}\text { baseline } \\
\text { model }\end{array}$ & $\begin{array}{l}\text { with SAT } \\
\text { in training }\end{array}$ & $\begin{array}{l}\text { with MLLR } \\
\text { in evaluation }\end{array}$ & $\begin{array}{l}\text { combined } \\
\text { schemes }\end{array}$ \\
\hline \multirow{4}{*}{$\begin{array}{c}\text { GI } \\
\text { model }\end{array}$} & \multirow[b]{2}{*}{$\sqrt{ }$} & $\sqrt{ }$ & GI+SAT+MLLR \\
\hline & & $x$ & N/A \\
\hline & \multirow[b]{2}{*}{$\times$} & $\sqrt{ }$ & GI+MLLR \\
\hline & & $x$ & GI \\
\hline \multirow{4}{*}{$\begin{array}{l}\text { GD } \\
\text { model }\end{array}$} & \multirow[b]{2}{*}{$\sqrt{ }$} & $\sqrt{ }$ & GD+SAT+MLLR \\
\hline & & $x$ & N/A \\
\hline & \multirow[b]{2}{*}{$\times$} & $\sqrt{ }$ & GD+MLLR \\
\hline & & $x$ & GD \\
\hline
\end{tabular}

Table .1 Schemes to improve model canonicity (x means without)

As showed in Table 1, two baselines: gender independent (GI) model and gender dependent (GD) model, plus two optional strategies: SAT in training and MLLR in evaluation, overall, six kinds of combined scheme are investigated in the experiment part.

\section{DATABASE}

Our database is carefully designed in order to be consistent with official PSC and the detailed descriptions are showed as follows:

There are totally 140 (70 male and 70 female) speakers. Among them, 100 (50 males and 50 females) speakers with certified standard pronunciations are selected to train the gold standard model. The rest 40 speakers whose pronunciation qualities varied from very bad with strong accents to standard are reserved as the testing data for both subjective and automatic evaluations.

Each speaker pronounces two full sets (Set A and Set B) and each set consists of 4 parts: 100 single syllablic words (Part1), 49 multi-syllablic words consisting of 100 syllables (Part2), a reading paragraph with approximately 400 syllables (Part3) and a spontaneous monologue (about 3 minutes) of roughly 400 syllables. Both A and B are randomly generated from large testing corpora.

Obviously, reading speech with more syllables can be used to assess the pronunciation proficiency of a speaker more reliably and accurately. Thereby, Part3 is selected in this study to verify the proposed approach.

\section{EXPERIMENT}

In this section, we first introduce the human scoring in terms of intra-rater and inter-rater correlation. Then we describe the machine scoring based on the schemes proposed in Section 2.

\subsection{Human scoring}

To obtain the human scores, three expert raters with national certificate are invited to score each part of each speaker. For Part3, each expert gives a general subjective score from several aspects such as number of mispronunciations, fluency, timing and so on. All human results reported here are based on Part3.

As a reference for machine scoring, it's very important to check the consistency and reliability from human scoring. Intra- 
rater and inter-rater correlation that measure the consistency within a rater and the agreement between raters are studied respectively.

\subsubsection{Intra-rater correlation}

Each target pronounced two sets: Set A and Set B and they are scored by each expert. Both Set A and Set B are randomly selected from large corpora and there is no significant difference on difficulties and therefore target's proficiency based on either A or $B$ should be consistent. Therefore, the consistency of a rater called intra-rater correlation can be calculated based on the scores between A and B (40 scores of each set) from the same rater.

As it is clear from Table 1, the consistency of different rater varies greatly from 0.779 to 0.919 . It shows in some degree how the subjective assessment on the same testing speaker even given by experts changes with the time.

Table.2 Intra-rater correlation

\begin{tabular}{|c|c|c|c|c|}
\hline Raters index & Rater1 & Rater2 & Rater3 & Average \\
\hline Correlation & 0.919 & 0.779 & 0.794 & 0.831 \\
\hline
\end{tabular}

\subsubsection{Inter-rater correlation}

The agreement between raters called inter-rater correlation is calculated from the correlation between scores of two different raters ( 80 scores on $\mathrm{A}$ and $\mathrm{B}$ given by each rater). From the result in Table 2, we can find that inter-rater correlation is still not high considerably. It means that the assessment disagreement on the same materials from same speakers given by different raters often occurs. Nevertheless, it is the upper bound for the machine scoring.

Table.3 Inter-rater correlation

\begin{tabular}{|c|c|c|c|}
\hline Correlation & Rater1 & Rater2 & Rater3 \\
\hline Rater1 & 1.0 & 0.756 & 0.784 \\
\hline Rater2 & & 1.0 & 0.802 \\
\hline Rater3 & & 1.0 \\
\hline \multicolumn{4}{|c|}{ Average: 0.781} \\
\hline
\end{tabular}

\subsection{Machine scoring}

As we known, Mandarin is a tonal language. Tones are more difficult to be pronounced correctly because they are much easily influenced by the dialect of the speaker. Therefore, tone mispronunciation is an important factor in PSC. Studies have indicated that F0 related features can greatly improve tone recognition accuracy, but how to deal with the unvoiced region without observation of $\mathrm{F} 0$ is always a big problem.

Multi-space distribution (MSD) approach, first proposed by Tokuda [7] for speech synthesis, can deal with the discontinuity of F0 elegantly and achieve good performance in tonal language speech recognition and tone mispronunciation detection [8]. In our experiment, MSD-HMM consisting of 184 phones is adopted. The acoustic feature vector contains 39-dimension spectral features (MFCC_E_D_A_Z) and 5-dimension F0 related features.

The utterances are segmented into syllables in order to get better performance. Tri-phone MSD-HMM is adopted to generate the boundary of each syllable using forced alignment. The averaged 3 rater's scores on all parts is calculated as the referenced scores for machine scoring, which can provide most reliable approximation to the ground-truth of the pronunciation proficiency for testing speakers. Therefore, there are totally 80 scores as the referenced scores by lining up both Set A and Set B.

\subsubsection{Effect of MLLR in APE}

MLLR can eliminate the mismatch between the target model and the adaptation data. Regression tree is used here to tie the regression matrices of MLLR. The effect of MLLR in APE with 1 leaf node of regression tree is shown is Table 4. The details of the correlation improving with the amount of evaluated syllable can be found in Figure 2. These GOP scores are all calculated directly at the syllable level. As it is clear in Table 4, the GD model makes better performance than the GI model because GD model removes the mismatch from gender. In addition to the gender variability removed by GD, MLLR can make further improvement. Meanwhile, the results based on GD+MLLR is still a little better than that on GI+MLLR shows that MLLR can learn some more speaker-specific characteristics in addition to the gender factors already rendered by GD model.

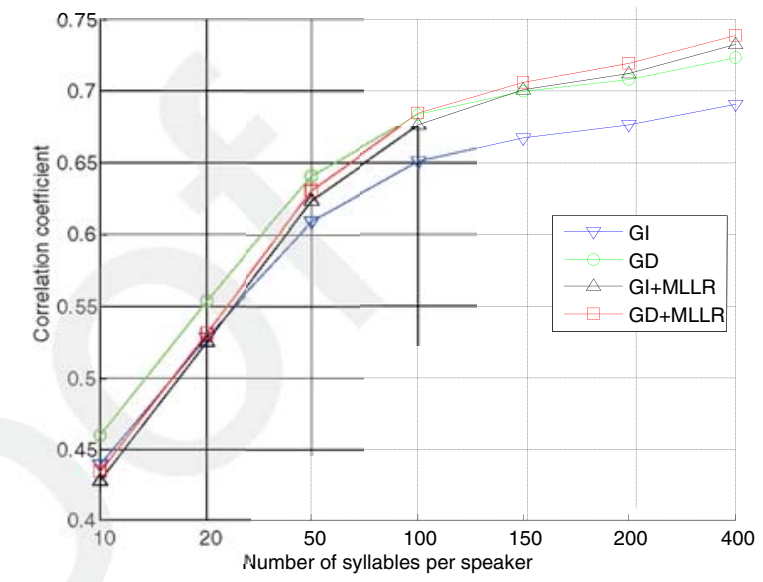

Fig.2 Correlation for different numbers of syllables per speaker

\subsubsection{Effect of $S A T$ in $A P E$}

As shown by above section, GD improves the performance a lot by removing the gender difference. But the target model is still affected by the variation existed in speakers even within the same gender. To normalize the training data further, SAT with CMLLR is adopted in our experiment to estimate a new model with smaller variance. Regression tree is used to tie the regression matrices of CMLLR. After estimating the new "neutral" model, MLLR with 1 leaf node or global transformation is used to adapt the testing data further. The comparison result with/without SAT is summarized in Table 4. The effect of the amount of the leaf nodes for regression tree of CMLLR is showed in Figure 3.

Table.4 Effect of SAT/MLLR for correlation between human and machine scores

\begin{tabular}{|c|c|c|c|}
\hline Schemes & $\begin{array}{c}\text { w/o SAT } \\
\text { w/o MLLR }\end{array}$ & $\begin{array}{c}\text { w/o SAT } \\
\text { with MLLR }\end{array}$ & $\begin{array}{c}\text { with SAT } \\
\text { with MLLR }\end{array}$ \\
\hline GI & 0.691 & 0.733 & 0.741 \\
\hline GD & 0.724 & 0.739 & 0.751 \\
\hline
\end{tabular}

By comparing the last two columns in Table 4, it not only shows the effectiveness of SAT, but also shows the additivity of the proposed schemes such as GD, SAT and MLLR. Generally speaking for CMLLR in SAT, with more leaf nodes, the better normalization is realized and better correlation is achieved. 


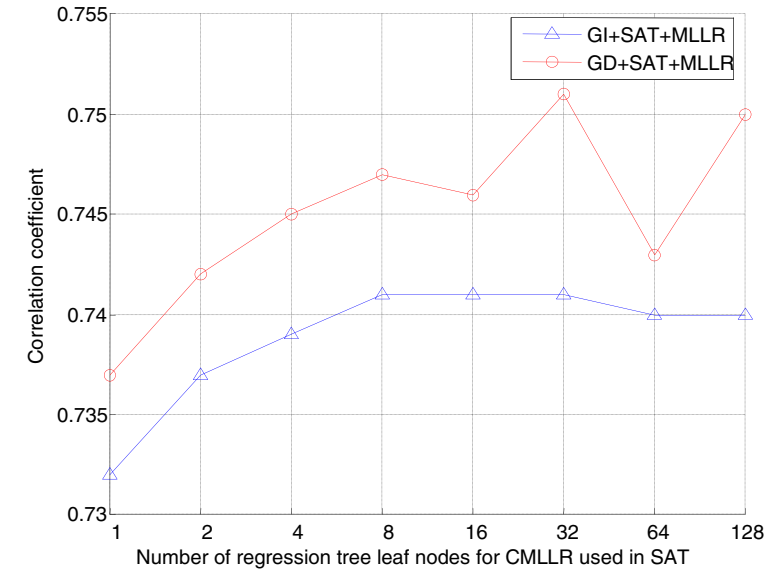

Fig.3 Correlation based on different numbers of regression leaf nodes for CMLLR used in SAT

\subsubsection{Correlation at phone-level based on SAT+MLLR}

The above results are computed at syllable level. As modeling based on phone unit has better resolution than that based on syllables, we also tested the phone level correlation. The results based on GD+SAT+MLLR at syllable and phone level are compared in Table 5. It shows that GOP based on phone log-PP score achieves better performance than that based on syllable one. Furthermore, GOPs based on weighted phones Log-PP by their duration can improve the correlation slightly further. The improvement may due to the fact that more reliable computation of log-PP can be obtained by weighting longer phone units more. In Figure 4, the difference of correlation between phones and syllable level log-PP decreases when more tested syllables per speaker are used.

Table.5 Correlation between human and machine scores

\begin{tabular}{|c|c|}
\hline GOPs & Correlation \\
\hline Syllable level log-PP & 0.751 \\
\hline Averaged phones log-PP & 0.758 \\
\hline Weighted phones log-PP & 0.762 \\
\hline
\end{tabular}

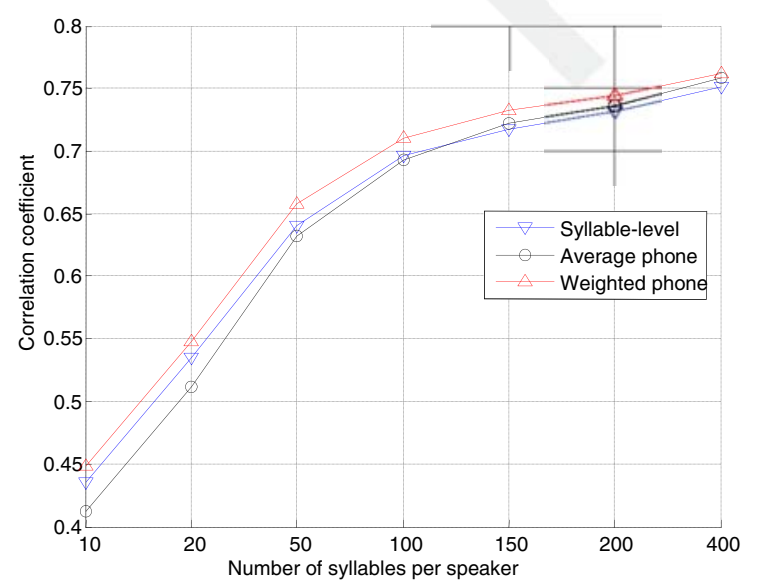

Fig.4 Correlation based on syllable and phone level GOPs for different numbers of syllables per speaker

\subsection{The consistency of machine scoring}

In addition to higher correlation expected for machine scoring, keeping consistent during the evaluation is also critical. Consistency calculation in APE uses the same manner as intra-rater correlation but replaces human scores with the machine scores based on weighted phone log-PP with scheme GD+SAT+MLLR. The comparisons between human scoring and automatic scoring are summarized in Table 6. The performance of human scoring in Table 6 means the agreement between raters, or inter-correlation.

Table.6 Summary of comparison between human-machine scoring

\begin{tabular}{|c|c|c|}
\hline & Human scoring & Machine scoring \\
\hline Consistency & 0.831 & 0.921 \\
\hline Performance & 0.781 & 0.762 \\
\hline
\end{tabular}

As shown in the Table 6, the consistency of machine scoring of 0.92 is much higher than 0.83 , that of human scoring. It indicates that machine scoring is more consistent than human experts. This is conceivable since human experts can be affected by fatigues and other emotional or physical factors. The fact that machine scoring nearly approaches the correlation upper bound among different human raters shows the merit of our proposed SAT and MLLR adaptation.

\section{CONCLUSION}

In addition to searching for a better GOP measure, to build a speech recognition model invariant to the test speaker's voice characteristics but sensitive to pronunciation accuracy is the main focus of this study. We investigate two strategies to improve the APE model: SAT-based model training to build an evaluation model insensitive to speaker specific variability; MLLR-based speaker adaptation to reduce the mismatch between the test speaker's characteristics and APE model. Experiments show that the proposed approach achieves a high correlation of 0.76 , a value very close to 0.78 between human experts. It is also observed that the proposed APE achieves much better consistency with a correlation of 0.92 than the value of 0.83 among human experts.

\section{REFERENCES}

[1] Zheng, J., Huang, C., Chu, M., Soong, F. K., Ye, W., "Generalized Segment Posterior Probability for Automatic Mandarin Pronunciation Evaluation”, in Proc. ICASSP, pp. IV201-204, Hawaii, USA, 2007.

[2] Witt, S., M, "Use of Speech recognition in Computer assisted Language Learning", PhD Thesis, University of Cambridge, 1999.

[3] Anastasakos, T., McDonough, J., Schwartz, R. \& Makhoul, J. "A compact model for speaker-adaptive training", in Proc ICSLP, Philadelphia, pp. 1137-1140, 1996.

[4] Giuliani, D., Gerosa, M., Brugnara, F., "Improved automatic speech recognition through speaker normalization", computer speech and language, 20, pp.107-123, 2006.

[5] Gales, M.J.F, "Maximum likelihood linear transformations for HMMbased speech recognition", Computer Speech and Language, 12, pp.75-98, 1998.

[6] Cucchiarini, C., Strik, H., Boves, L., "Different aspects of expert pronunciation quality ratings and their relation to scores produced by speech recognition algorithms", Speech Communication, 30 (2-3), pp. 109$119,2000$.

[7] Tokuda, K., Masuko, T., Miyazaki, N., and Kobayashi, T., "Multi-space Probability Distribution HMM", IEICE Trans. Inf. \& Syst., E85-D(3): pp. 455-464, 2002.

[8] Zhang, L., Huang, C., Chu, M., Soong, F. K. "Automatic detection of tone mispronunciation in Mandarin Chinese", in Proc. ISCSLP, LNAI 4272, pp. 590-601, Springer, 2006.

[9] Franco, H., Neumeyer, L., Y, Kim and O. Ronen, "Automatic pronunciation scoring for language instruction," in Proc. ICASSP, pp. 1471-1474, Munich, Germany, April 1997. 